\title{
Evaluation Serum Copper Levels in Preeclampsia and Healthy Pregnant Women
}

Baral Na, Mishu FA ${ }^{b}$, Haque $\mathrm{R}^{\mathrm{a}}$

\begin{abstract}
Background: Preeclampsia is a fatal medical disorder of pregnancy. It has been associated with adverse course and outcome of the pregnancy resulting in increased maternal and infant mortality and morbidity. As the pathogenesis of preeclampsia is not completely understood, prevention remains a complex issue. In recent times, there has been an increasing prevalence in the incidence of preeclampsia globally. The aim of this study is to determine the states of the copper in pregnant women with preeclampsia.

Methods: This case-control study was carried out in the Department of Biochemistry, Mymensingh Medical College, Mymensingh, from July 2013 to June 2014. The subjects were selected on the basis of inclusion and exclusion criteria by purposive method. This study included 73 patients with preeclampsia as case (Gr.I) classified into two subgroups according to the gestational age: Gr.I(a); preeclampsia in the second trimester $(n=35)$, and Gr.I(b); preeclampsia in the third trimester $(n=38)$. Copper levels were analyzed and results were compared with 73 apparently healthy pregnant control (Gr.II), and the corresponding gestational age subgroups [Gr.II(a); normal pregnancy of second trimester $(n=35)$ and Gr.II(b), normal pregnancy of third trimester $(n=38)]$.

Results: The mean serum copper level was significantly $(p<0.001)$ lower in case group than that of control group. Similar extent of reduction was observed in serum copper level at different gestational age groups of preeclamptic women when compared with corresponding control groups.
\end{abstract}

Conclusion: Estimation of serum copper level should be incorporated in pre eclamptic patients for prevention of complications.

Keywords: Copper, Pregnancy, Preeclampsia.

(BIRDEM Med J 2019; 9(1): 18-22)

\section{Introduction}

Preeclampsia is a multisystem disorder of unknown etiology associated with increased maternal and fetal morbidity and mortality. Preeclampsia is the third common cause of maternal death in the world. ${ }^{9}$

1. Dr. Nilima Baral, Dr. Rezwana Haque, Department of Biochemistry, Uttara Adhunik Medical College, Uttara, Dhaka, Bangladesh.

2. Dr. Farzana Akonjee Mishu, Department of Physiology and Molecular Biology. Bangladesh Institute of Research \& Rehabilitation in Diabetes, Endocrine \& Metabolic Disorders (BIRDEM) Dhaka, Bangladesh.

Correspondence: Dr. Nilima Baral, Assistant Professor, Biochemistry, Uttara Adhunik Medical College \& Hospital, Uttara, Dhaka. Email: nilima.barallotus @gmail.com

Received: January 18, 2018

Accepted: October 31, 2018
Preeclampsia stands next to hemorrhage and embolism in pregnancy related cause of death. The world Health Organization estimates that globally between 50000 and 75000 women die of this condition each Year $^{5}$. In Bangladesh $16 \%$ of maternal deaths are caused by Preeclampsia and eclampsia ${ }^{8}$.

Preeclampsia is defined as hypertension associated with proteinuria that arise for the first time after the 20th week of gestation in a previously normotensive woman. Proteinuria resolve completely by the 6th postpartum week. ${ }^{1}$ High blood pressure, swelling of lower extremities, protein in the urine, sudden weight gain, headaches and changes in vision are the symptoms of pre eclampsia. Increased blood pressure $\geq 140 \mathrm{~mm} \mathrm{Hg}$ systolic or $\geq 90 \mathrm{~mm} \mathrm{Hg}$ diastolic on two separate readings taken at least four to six hours apart after 20 
weeks gestation in an individual with previously normal blood pressure is the important sign of preeclampsia. ${ }^{2}$

Normal pregnancy is associated with profound changes in maternal homeostasis. The pathophysiology of preeclampsia is thought to represent a defective response to the physiologic demands of normal pregnancy ${ }^{10}$. Different researchers have demonstrated that macro and micro nutrients are essential for the optimum development of fetus. Among the micronutrients, $\mathrm{Cu}$ is important, especially early in the life for the development and maintenance of fetal organs and tissues. Copper is an essential micronutrients. During pregnancy, plasma copper concentrations significantly increase, returning to normal values after delivery. ${ }^{13}$ It is involved in the function of several coenzymes that are essential for life. It also shows antioxidant activity ${ }^{15}$ The fetus is fully dependent on the maternal copper supply. Its deficiency during embryonic and fetal development can result in numerous gross structural and biochemical abnormalities it is estimated that more than $50 \%$ of human conceptions fail to implant, and of those implanting, approximately $30 \%$ fail to reach term. Deficiencies of $\mathrm{Cu}$ have been implicated in infertility, pregnancy wastage, congenital abnormalities, still birth and low birth weight ${ }^{7,8}$

The study was designed to evaluate the copper status in pregnant women with preeclampsia and also compare with the normal healthy pregnant women.

\section{Methods}

This case-control study was carried out from July 2013 to June 2014 in the Biochemistry department of Mymensingh Medical College Hospital, Mymensingh. Total 146 subjects were selected by purposive method on the basis of eligibility criteria. Out of them 73 clinically diagnosed preeclampsia patient were case, (Gr.I) and 73 normal pregnant women were control (Gr.II). The exclusion criteria used for both case and control were women having preexisting of hypertension, pregnancy less than 20 weeks of gestation, acute liver diseases, cardiovascular disease, diabetes mellitus and other systemic disorders.

The 73 patients of both cases and controls were further classified into two subgroups according to the gestational age:

- Preeclampsia in the second trimester Gr.I(a): $(n=35)$

- Preeclampsia in the third trimester Gr.I(b): $(n=38)$

- Normal pregnancy in the second trimester Gr.II(a): $(n=35)$
- Normal pregnancy in the third trimester Gr.II(b): $(\mathrm{n}=38)$

Data were recorded by using a pre-designed format of data collection sheet. Informed written consent was obtained from all participants. Blood samples were collected and analyzed for investigation. Diabetes Mellitus and liver diseases were excluded by fasting blood sugar and serum ALT respectively, Hypertension and any kind of endocrine disorder were excluded by taking history and clinical examination. Demographic, dietary and medical information and other relevant data were collected and recorded in a preformed data sheet. For laboratory investigations, $5 \mathrm{ml}$ blood was collected, processed and preserved for estimation of different biochemical parameters.

Serum copper was determined by sensitive, direct colorimetric assay for copper in serum ${ }^{16}$.The data were processed and analyzed by computer software SPSS (Statistical Package for Social Science) version 14.0. Student's unpaired t- test was used to analyze the data between groups. Values are expressed as Mean \pm SD. For analytical tests, the confidence limit was $95 \%$ and $\mathrm{p}<0.05$ was taken as level of significance.

\section{Results}

Different variables of the subjects were being analyzed and compared between case and control. Some physical aspects such as systolic blood pressure, diastolic blood pressure and age are shown in Table I. The study showed that edema was common in patient with preeclampsia. Among 73 cases, 64 (87.67\%) were suffering from edema and $9(12.33 \%)$ were free from edema. In control group none had edema. Out of 73 case, 59 (80.82\%) were primigravida and $14(19.18 \%)$ were multigravida.

Table I Characteristics in the study population

\begin{tabular}{lccc}
\hline Variables & $\begin{array}{c}\text { Mean } \pm \text { SD } \\
\text { (case) }\end{array}$ & $\begin{array}{c}\text { Mean } \pm \text { SD } \\
\text { (control) }\end{array}$ & $\begin{array}{c}\mathrm{p} \\
\text { value }\end{array}$ \\
\hline Age (years) & $24 \pm 2.89$ & $25 \pm 2.73$ & $\mathrm{p}>0.05$ \\
DBP(mm of Hg) & $100 \pm 8.55$ & $75 \pm 5.36$ & $\mathrm{p}<0.001$ \\
SBP (mm of Hg) & $152 \pm 7.41$ & $118 \pm 4.62$ & $\mathrm{p}<0.001$ \\
\hline
\end{tabular}

$\mathrm{p}<0.001=$ highly significant; $\mathrm{p}>0.05=$ not significant

$\mathrm{SD}=$ Standard deviation

$\mathrm{SBP}=$ Systolic blood pressure

$\mathrm{DBP}=$ Diastolic blood pressure 
Preeclampsia Patients had significantly $(\mathrm{p}<0.001)$ low cu level than healthy pregnant women. Also highly significant decrease of serum copper level was again observed when case subgroups Gr.I(a) and Gr.I(b) were compared with corresponding control Gr.II(a) and Gr.II(b) respectively $(p<0.001)$. Analysis of mean serum copper levels of study population were presented in Table II.

Table II Comparison of mean serum copper levels in the study population

\begin{tabular}{lcc}
\hline Group & Mean \pm SD $($ ig $/ \mathrm{dl})$ & $\mathrm{p}$ value \\
\hline Gr.I & $147.47 \pm 25.93$ & $\mathrm{p}<0.001$ \\
Gr.II & $200.36 \pm 38.67$ & \\
Gr.I(a) & $152.86 \pm 25.73$ & $\mathrm{p}<0.001$ \\
Gr.II(a) & $196.46 \pm 45.18$ & \\
Gr.I(b) & $142.50 \pm 25.43$ & $\mathrm{p}<0.001$ \\
Gr.II(b) & $203.95 \pm 31.73$ & \\
\hline
\end{tabular}

$\mathrm{p}<0.001=$ Highly significant

Gr.I = Case (Preeclampsia patient)

Gr.II $=$ Control (Normal pregnancy)

Gr.I(a) $=$ Preeclampsia in the second trimester

Gr.I(b) $=$ Preeclampsia in the third trimester

Gr.II(a) $=$ Normal pregnancy in the second trimester

Gr.II(b) $=$ Normal pregnancy in the third trimester

$\mathrm{SD}=$ Standard deviation

The difference in mean serum copper levels in relation to gestational age in both case and control subgroups were not significant $(\mathrm{p}>0.05)$. The mean values of serum copper levels were $152.86 \pm 25.733,14.50 \pm 25.43,196.46 \pm 45.18$ and $203.95 \pm 31.73 \mu \mathrm{g} / \mathrm{dl}$ for the Gr.I(a), Gr.I(b), Gr.II(a) and Gr.II (b) groups respectively. They were presented in Figure1.

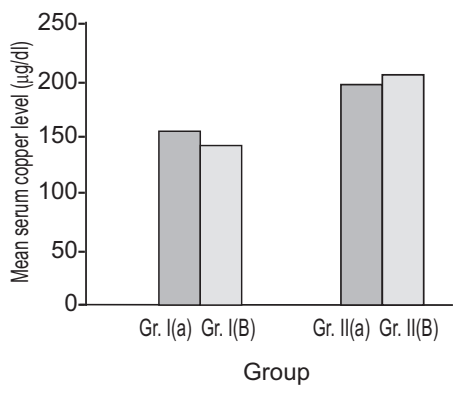

Figure 1 Mean serum copper levels in the study population according to gestational age Gr.I(a) $=$ Preeclampsia in the second trimester Gr.I(b) = Preeclampsia in the third trimester Gr.II(a) $=$ Normal pregnancy in the second trimester Gr.II(b) = Normal pregnancy in the third trimester

\section{Discussion}

This study showed that preeclampsia is more common in primigravida pregnant mother than that of multigravida pregnant mother. Previous study showed incidence of preeclampsia in primigravida is about $10 \%$ and in multigravidae about $5 \% .{ }^{4}$

In this study, serum copper level is lower in preeclamptic patient than normal pregnant woman. This difference is highly significant $(p<0.001)$. This result is consistent with some previously conducted studies. They found significant difference in serum copper level in preeclamptic patient when compare with healthy pregnant . 1,2,4,5,12 But few study show conflicting result with this study. They observed that serum copper level is higher in preeclampsia patient than normal pregnancy group. Cu gave a negative association in preeclampsia. ${ }^{17}$ This may contribute to oxidative stress, later in pregnancy, in those women that go on to develop preeclampsia ${ }^{15}$. Another study concludes that there is no significant difference in mean serum copper level in between preeclampsia and normal pregnant group ${ }^{18}$.

Serum copper changes with the time of gestation. It has been observed that maternal copper level is elevated from the luteal phase of the pregnancy and rises with the progression of pregnancy. At term total serum concentration of copper in the mother is twice than that of normal level ${ }^{13}$. The possible causes of the changes are due to the hormonal, metabolic and enzymatic changes in pregnancy. The physiological increase in copper concentration is partly associated with estrogen induction of copper caring protein ${ }^{18}$.

Though the copper level is increased, the percentage of the free copper is not significantly changed. It is caused by binding of copper with copper binding protein, ceruloplasmin which is increased. Ceruloplasmin catalyzes the conversion of ferric ion to its ferrous form and promotes the absorption of iron from the gastrointestinal tract. Increase level of ceruloplasmin is found in pregnancy with preeclampsia; this positively correlated with increase production of antioxidant protein in response to increase lipid per oxidation ${ }^{19}$. The role of oxidative stress or excessive lipid per oxidation has been implicated in preeclampsia. Low level of copper in preeclamptic women may be 
associated with impairment of the cell antioxidant capacity and oxidant/antioxidant balance. The imbalance between antioxidant enzyme activities and pro-oxidant production leading to atypical biochemical changes resulting in preeclampsia ${ }^{6}$.

Micronutrients such as copper play a vital role in metabolic pathways. The many biochemical reaction and particularly those with oxido-reduction capacities entirely associated with the presence of enough micronutrient. The enzymes which utilize copper as their cofactors can consume oxygen as in oxido-reduction pathways. This area of metabolic pathways are verified, but chemical energy production in human within inner mitochondrial membrane and detoxification of many drugs and toxin within the liver entirely dependent on those enzyme and metaloprotein comprising copper as the core subsequent ${ }^{20}$. In pregnancy, the processes of implantation, proliferation, differentiation and trophoblast invasion produce reactive oxygen species (ROS), while in preeclampsia, lipid peroxidation also yielding ROS is uncontrolled. It is thought that preeclampsia is associated with an imbalance of increased lipid peroxides and decreased antioxidants ${ }^{4}$.

Different study designs, different techniques of analysis, small samples and different population characteristics such as race, culture, eating habits and geographical regions are possible explanations for the different results of this study with other studies.

\section{Conclusion}

The present study conclude that preeclampsia in both second and third trimester were associated with low serum copper level when compare with healthy pregnant women. Therefore it may be recommended that estimation of copper level in antenatal period should be carried out for reduce the incidence of preeclampsia and management of preeclampsia.

Conflict of interest: Nothing to declare.

\section{References}

1. Al-Shalah HH, Al-Hilli MM, Hasan MA. The Association of Serum Iron, Zinc, and Copper Levels with Preeclampsia. Medical Journal of Babylon 2016; 12:4, doi:1812-156X-12-4.

2. Rafeeinia A, Tabandeh A, Khajeniazi S, Marjani AJ. Serum copper, zinc and lipid peroxidation in pregnant women with preeclampsia in Gorgan. Open Biochem J 2014; 8: 83-88.
3. Mustafa, Reem, Ahmed, Sana, Gupta, Anu, Venuto, Rocco C. A Comprehensive Review of Hypertension in Pregnancy. Journal of Pregnancy 2012; 10: 1-19.

4. Noura AJ, Hajera T, Mir NA, Mohammed AQ, Farah AK, May AR. Correlation between serum trace elements and risk of preeclampsia: A case controlled study in Riyadh, Saudi Arabia 2015;doi:10.1016/j.sjbs.2015.02.009.

5. Basima SA, Alaa MS, Nabras NH. The role of Trace Elements (Zinc, Copper, Magnesium and Calcium) in Pregnant Women with Preeclampsia in third trimester and fetal cord blood after delivery. Copyright $\subset 2014$ BS. Creative Commons Attribution License (http://creativecommons. org/licenses/by/ 2.0)

6. Ugwuja EI, Ejikeme BN, Ugwu NC, Obeka NC, Akubugwo EI, Obidoa O. Comparison of plasma copper, iron and zinc levels in hypertensive and non-hypertensive pregnant women in Abakaliki, South Eastern Nigeria. Pak J Nutr 2010;9(12):1136-40. DOI via Crossref.

7. Akhtar S, Begum S and Ferdousi S. Calcium and Zinc Deficiency in Preeclamptic Women. J. Bangladesh Soc Physio 2011; 6 (2): 94-99.

8. Sarwar MS, Ahmed S, Ullah MS, Kabir H, Rahaman GKM, Hasnat A and Islam MS. Comparative Study of Serum Zinc, Copper, Manganese, and Iron in Preeclampti Pregnant Women. Biol Trace Elem Res 2013; 154: 14 - 20.

9. Homeira V, Maryam D and Seyed AH. Serum concentration of calcium, magnesium and zinc in normotensive versus preeclampsia pregnant women: A descriptive study in women of Kerman province of Iran. Iran J Reprod Med 2015; 13(1): $23-26$.

10. Steegers, Eric AP, Von Dadelszen, Peter, Duvekot, Johannes J, Pijnenborg and Robert. Pre-eclampsia. The Lancet 2010; 376 (9741): 631-644.

11. Al-Jameil N, Tabassum H, Al-Mayouf H, Aljohar HI, Alenzi $\mathrm{ND}$, Hijazy SM et al. Analysis of serum trace elementscopper, manganese and zinc in preeclamptic pregnant women by inductively coupled plasma optical emission spectrometry: A prospective case controlled study in Riyadh, Saudi Arabia. Int J Clin Exp Pathol 2014; 7: 1900-1910.

12. Kanagal DV, Rajesh A, Rao K, Devi UH, Shetty H, Kumari $\mathrm{S}$, et al. Levels of serum calcium and magnesium in preeclamptic and normal pregnancy: A study from Coastal India. J Clin Diagn Res. 2014;8:1-4.

13. Liu J, Yang H and Shi H. Blood copper, zinc, calcium, and magnesium levels during different duration of pregnancy in Chinese. Biological Trace Element Research. 2010;135, (1): 31-37.

14. Shakour-Shahabi L, Abbasali-Zadeh S and Rashtchi-Zadeh N. Serum level and antioxidant activity of ceruloplasmin in preeclampsia. Pakistan Journal of Biological Sciences. 2010; (13): 621-27. 
15. Mistry HD, Gill CA, Kurlak LO, Seed PT, Hesketh JE, Méplan $\mathrm{C}$ et al. Association between maternal micronutrient status, oxidative stress, and common genetic variants in antioxidant enzymes at 15 weeks $\times$ gestation in nulliparous women who subsequently develop preeclampsia. Free Radic Biol Med. 2015;78:147-55. doi: 10.1016/j.freeradbiomed.2014.10.580.

16. Abe A, Yamashita S and Noma A. Sensitive, direct colorimetric assay for copper in serum. Clin Chem. 1989; 35(4):552-4.

17. Ahsan T, Banu S, Nahar Q, Ahsan, M, Khan MN and Islam $\mathrm{SN}$. Serum trace elements levels in preeclampsia and eclampsia: Correlation with the pregnancy disorder. Biol. Trace Elem. 2013; 152: 327-332.

18. Faisal Gh And Al-Rubaye. Trace Elements Homeostasis in Preeclampsia. Iraqi J Med Sci. 2009; 7 (2): 116-123.

19. Farzin L and Sajadi F. Comparison of serum trace element levels in patients with without pre-eclampsia. J Res Med Sci. 2012; 17 (10): 938-941.

20. Bakhshande, Nosrat S, Ghaemi EA, Ahmadi A, Behnampou $\mathrm{N}$, Marjani A et al. Maternal Serum Copper Concentration in Premature Rupture of Membrane:A case control study. Journal of Biological science. 2014;14:73-76. DOI: 10.3923/ jbs.2014.73.76. 